\title{
Failure or success of search strategies to identify adverse effects of medical devices: a feasibility study using a systematic review
}

Su Golder ${ }^{*}$, Kath Wright and Mark Rodgers

\begin{abstract}
Background: Research has indicated that adverse effects terms are increasingly prevalent in the title, abstract or indexing terms of articles that contain adverse drug effects data in MEDLINE and Embase. However, it is unknown whether adverse effects terms are present in the database records of articles that contain adverse effects data of medical devices, and thus, to what extent the development of an adverse effects search filter for medical devices may be feasible.
\end{abstract}

Methods: A case study systematic review of a medical device was selected. The included studies from a systematic review of the safety of recombinant human bone morphogenetic protein-2 (rhBMP-2) for spinal fusion were used in the analysis. For each included study, the corresponding database record on MEDLINE and Embase was assessed to measure the presence or absence of adverse effects terms in the title, abstract or indexing. The performance of each potential adverse effects search term was also measured and compared.

Results: There were 82 publications (49 studies) included in the systematic review with 51 of these indexed on MEDLINE and 55 on Embase. Ninety-four percent (48/51) of the records on MEDLINE and 95\% (52/55) of the records on Embase contained at least one adverse effects related search term. The wide variety of adverse effects terms included in the title, abstract or indexing of bibliographic records, and the lack of any individual high-performing search terms suggests that a combination of terms in different fields is required to identify adverse effects of medical devices. In addition, the most successful search terms differed from the most successful terms for identifying adverse drug effects.

Conclusions: The search filters currently available for adverse drug effects are not necessarily useful for searching adverse effects data of medical devices. The presence of adverse effects terms in the bibliographic records of articles on medical devices, however, indicates that combinations of adverse effects search terms may be useful in search strategies in MEDLINE and Embase. The results, therefore, suggest that not only a search filter for the adverse effects of medical devices is feasible, but also that it should be a research priority.

Keywords: Adverse effects, Systematic reviews, Meta-analysis, Information storage and retrieval, Bibliographic databases

\footnotetext{
* Correspondence: su.golder@york.ac.uk

Centre for Reviews and Dissemination (CRD), University of York, York YO10
} $5 \mathrm{DD}, \mathrm{UK}$

\section{Biomed Central}

(C) 2014 Golder et al.; licensee BioMed Central Ltd. This is an Open Access article distributed under the terms of the Creative Commons Attribution License (http://creativecommons.org/licenses/by/4.0), which permits unrestricted use, distribution, and reproduction in any medium, provided the original work is properly credited. The Creative Commons Public Domain Dedication waiver (http://creativecommons.org/publicdomain/zero/1.0/) applies to the data made available in this article unless otherwise stated. 


\section{Background}

Adverse effects are an important consideration in decisionmaking by patients, clinicians and other health care professionals, policy makers and regulators. An adverse effect is a 'harmful or undesirable outcome that occurs during or after the use of a drug or intervention for which there is at least reasonable possibility of a causal relation' [1]. Although adverse effects can occur with any intervention, methodological research into how to find information on adverse effects has, to date, concentrated on identifying adverse effects of drug interventions [2]. The adverse effects of other interventions such as surgery, medical devices, diagnostic tests and physical interventions, however, are equally important. In particular, the importance of the adverse effects of medical devices has been highlighted recently in the press by articles on the potential harm from breast implants and hip prostheses. Although the regulatory process for medical devices is less stringent than for pharmaceutical interventions, the adverse effects of medical devices can be just as serious and can be an important factor in decisionmaking for health care professionals, policy makers and patients. Identifying the evidence on adverse effects for medical devices is, therefore, paramount to inform health care decisions.

Recent research has indicated that database searching using adverse effects terms will now retrieve the majority of articles on adverse drug effects (92\%) [3,4]. However, many of the terms that have proved useful are specific to the adverse effects of drug interventions (for example, adverse drug reaction, chemically induced, toxicity, drug effects, drug induced, drug monitoring and drug hypersensitivity). Similarly, the adverse effects search filters developed to date have also focused on identifying adverse drug effects [2].

A different approach may be required to searching for adverse effects of non-drug interventions, and this is confirmed by a recent study that tested the efficiency of existing search filters in identifying adverse effects papers related to medical devices. While these search filters usually obtain around $92 \%$ of the relevant articles in MEDLINE and $89 \%$ of the relevant articles in Embase, the same filters only retrieved $74 \%$ of the relevant articles related to medical devices in MEDLINE and 54\% in Embase [5].

The development of search filters for adverse effects of non-drug interventions and for medical devices, in particular, is therefore a key priority. The first stage of any search filter development should be to assess its feasibility. Search terms related to adverse effects need to be present in the bibliographic database records (in the title, abstract or keywords fields) in order for any article to be potentially retrievable by using adverse effects terms in the database search strategy.

In this case study, we explore the presence or absence of terms related to adverse effects in the bibliographic records of those articles included in a systematic review of a medical device. In addition, we present those terms that are the most useful for identifying the adverse effects of this case study medical device.

\section{Methods}

A case study systematic review of the safety of recombinant human bone morphogenetic protein-2 (rhBMP-2) was selected for analysis [6,7]. Here, rhBMP-2 is a medical device (collagen sponge) that is widely used as an alternative to iliac crest bone graft to promote fusion in spinal surgery. RhBMP-2 is licensed as a medical device with a pharmaceutical component and as with many other medical devices requires a surgical procedure for implementation.

This review was undertaken after a number of sources raised concerns about the safety of rhBMP-2: during the post-marketing period, several non-industry observational studies reported adverse events possibly associated with the use of rhBMP-2; the Food and Drug Administration (FDA) issued a public health notification of potentially life-threatening complications associated with swelling of the neck and throat tissue after rhBMP-2 use in the cervical spine; and a subsequent review of publicly available data suggested an increased risk of complications and adverse events for patients receiving rhBMP-2 that was 10 to 50 times higher than the original randomised controlled trial (RCT) estimates. These sources identified a number of specific adverse events of concern-some dependent upon spinal location-such as dysphagia, retrograde ejaculation, heterotopic bone formation and osteolysis. These possible adverse effects of rhBMP-2 have received much publicity, and a high-quality systematic review encompassing all the evidence was required to answer the many ambiguities. The Centre for Reviews and Dissemination (CRD) at the University of York was commissioned by Yale University to conduct such as a review. The systematic review of the safety of rhBMP-2 included a search of the following databases: BIOSIS Previews (1969-2008 only), CENTRAL, Embase, MEDLINE, PubMed, Science Citation Index (SCI), ClinicalTrials.gov, the Database of Abstracts of Reviews of Effects (DARE), the Health Technology Assessment (HTA) database and ToxFile. PubMed was selected in addition to MEDLINE as PubMed includes citations not included in MEDLINE as well as the MEDLINE database itself. In addition to database searching, various supplementary search methods were used that included reference checking; contacting authors of key papers; publishing a call for evidence in Spine Journal, The Back Letter newsletter and on the Internet and setting up automated 'current awareness' searches in Zetoc Alert from the British Library and in MEDLINE to notify us whenever new data matching our search criteria were loaded onto the databases. The broad search strategy 
contained just two facets: rhBMP-2 and spinal fusion with multiple synonyms, textwords and indexing terms used for each facet. No adverse effects terms were applied to the search strategy in order to try to maximise sensitivity and allow a prospective analysis of the potential performance of adverse effects terms if they had been included. A pragmatic decision was made to include disease terms (spinal fusion) although it was appreciated that this may have missed papers which referred to adverse effects of the intervention in other bone conditions, or where spinal fusion was not mentioned. No study design or date limits were applied, and the full search strategy is published elsewhere $[6,7]$.

\section{Inclusion criteria}

All studies (RCTs and observational studies) of more than ten adult participants that compared rhBMP-2 with any other spinal fusion technique and reported adverse effects were eligible for inclusion in the systematic review.

\section{Analysis}

The included references from this case study systematic review formed the basis of the analysis. This review contained 82 publications representing 49 studies. Of the 82 publications, 40 referred to 13 RCTs and one singlearmed study and the other 42 publications referred to 35 observational studies.

The first stage of the analysis was to check whether each paper was listed in MEDLINE or Embase. MEDLINE and Embase were selected as they are the most commonly searched databases in systematic reviews and have been evaluated for the availability of adverse drug effects terms in the previous research $[3,4,8]$. In order to ascertain whether each paper was contained in the databases, several iterations using author names and words from the title were used.

\section{Adverse effects terms in the database records}

For each database, the available papers were sorted according to the following criteria:

1. The authors mentioned terms synonymous with 'adverse effects' in the title or abstract, potentially enabling the papers to be found in an electronic search. Generic adverse effects terms, such as 'adverse events', 'side effects', 'complications', 'safe' and 'risk' were accepted.

2. The authors mentioned specific named adverse effects terms such as 'swelling', 'dysphagia' or 'blood loss' in the title or abstract.

3. The papers had been indexed (using subject headings or subheadings) with relevant terms for adverse effects, potentially enabling the papers to be found in an electronic search. Generic adverse effects terms were accepted on the basis that they could be considered synonymous with 'adverse effects'. Examples of included indexing terms are 'postoperative complications/' in MEDLINE and 'device safety/' in Embase. Examples of included subheadings are 'complications (co)' in MEDLINE and 'side effect (si)' in Embase.

4. The papers had been indexed with specific named adverse effects terms such as 'airway obstruction/' in MEDLINE or Embase, 'dysphagia/' in Embase, and 'bleeding/' in Embase.

\section{Results}

\section{Included records}

There were 51 of the 82 included references on MEDLINE (five were not identified at the time of the original searches) and 55 of the 82 included references on Embase (four were not identified at the time of the original searches). Seven of these included references from Embase were duplicated within in Embase, and for the purposes of this analysis, the duplicate records were not included.

\section{Adverse effects terms by search fields}

The percentage of relevant records with either generic or specific named adverse effects terms in the bibliographic records (title, abstract or indexing) in MEDLINE and Embase is presented in Table 1. The most commonly occurring adverse effects terms in the MEDLINE records were generic adverse effects terms in the abstract $(73 \%)$, followed by specific adverse effects terms in the abstract (61\%) and then generic adverse effects subheadings (51\%). In Embase, the most commonly occurring adverse effects terms were specific named adverse effects in the indexing (73\%), followed by generic adverse effects terms in the abstract $(71 \%)$ and then specific adverse effects terms in the abstract (67\%).

Overall, a search with generic adverse effects search terms would retrieve $88 \%$ of the available records in MEDLINE and $85 \%$ of the available records in Embase. Searching with specific named adverse effects terms would perform better in Embase than in MEDLINE retrieving 92\% of the records in Embase compared with 69\% in MEDLINE.

A search with both generic and specific named adverse effects in either MEDLINE or Embase would retrieve a high proportion of the records available in each of the databases (94\% and 95\%, respectively).

\section{Individual search terms}

The highest number of relevant records in MEDLINE was retrieved by the subheading 'adverse effects (ae)' (47\%), followed by 'complication' in the abstract (31\%), the indexing term 'postoperative complications/' (27\%), 'complications' in 


\section{Table 1 Adverse effects terms in the bibliographic records in MEDLINE or Embase}

\begin{tabular}{|c|c|c|}
\hline & $\begin{array}{l}\text { Relevant MEDLINE } \\
\text { records retrieved } \\
(N=51)\end{array}$ & $\begin{array}{l}\text { Relevant Embase } \\
\text { records retrieved } \\
(N=55)\end{array}$ \\
\hline \multicolumn{3}{|l|}{ Generic adverse effects terms } \\
\hline Title & $16 \%(8)$ & $\begin{array}{l}\text { 16\% (9 plus } 1 \\
\text { duplicate) }\end{array}$ \\
\hline Abstract & $73 \%(37)$ & $\begin{array}{l}71 \% \text { (39 plus } 4 \\
\text { duplicates) }\end{array}$ \\
\hline Title or abstract & $73 \%(37)$ & $\begin{array}{l}71 \% \text { (39 plus } 4 \\
\text { duplicates) }\end{array}$ \\
\hline Indexing & $31 \%(16)$ & $35 \%(19)$ \\
\hline Subheadings & $51 \%(26)$ & $62 \%(34)$ \\
\hline EMBASE section headings & NA & $16 \%(9)$ \\
\hline $\begin{array}{l}\text { Any generic adverse effects } \\
\text { terms }\end{array}$ & $88 \%(45)$ & $\begin{array}{l}85 \% \text { (47 plus } 5 \\
\text { duplicates) }\end{array}$ \\
\hline \multicolumn{3}{|l|}{ Specific adverse effects terms } \\
\hline Title & $18 \%(9)$ & $\begin{array}{l}20 \% \text { (11 plus } 1 \\
\text { duplicate) }\end{array}$ \\
\hline Abstract & $61 \%(31)$ & $\begin{array}{l}67 \% \text { (37 plus } 4 \\
\text { duplicates) }\end{array}$ \\
\hline Title or abstract & $61 \%(31)$ & $\begin{array}{l}67 \% \text { ( } 37 \text { plus } 4 \\
\text { duplicates) }\end{array}$ \\
\hline Indexing & $37 \%(19)$ & $\begin{array}{l}\text { 73\% (40 plus } 3 \\
\text { duplicates) }\end{array}$ \\
\hline $\begin{array}{l}\text { Any specific adverse effects } \\
\text { terms }\end{array}$ & $69 \%(35)$ & $\begin{array}{l}82 \% \text { ( } 45 \text { plus } 5 \\
\text { duplicates) }\end{array}$ \\
\hline $\begin{array}{l}\text { Any adverse effects terms in } \\
\text { any field }\end{array}$ & $94 \%(48)$ & $\begin{array}{l}\text { 95\% (52 plus } 5 \\
\text { duplicates) }\end{array}$ \\
\hline
\end{tabular}

the abstract (27\%), 'safety' in the abstract (27\%), 'safely' in the abstract (27\%) and then 'blood loss' in the abstract (20\%). A complete listing of the performance of individual search terms in MEDLINE is presented in Additional file 1: Table S1.

The highest number of relevant records in Embase was retrieved by the subheading 'complication (co)' (49\%), followed by the term 'complications' in the abstract (31\%); the indexing term 'pseudarthrosis/' (24\%); the subheading 'adverse drug reaction (ae)' (22\%); the terms 'blood loss', 'complications' and 'safety' in the abstract (18\%) and then the indexing terms 'bleeding/' and 'dysphagia/' (18\%). A complete listing of the performance of individual search terms in Embase is presented in Additional file 2: Table S2.

\section{Discussion}

This case study demonstrates the feasibility of developing a generic search filter for adverse effects of medical devices. Eighty-eight percent of MEDLINE records and 85\% of Embase records contained at least one generic searchable term for adverse effects. With the addition of specific named adverse effects terms, search performance could potentially reach 94\% in MEDLINE and 95\% in Embase.
The most successful individual adverse effects search terms varied from those identified in similar research using adverse drug effects case examples. There was a greater tendency for terms such as 'complication(s)', 'safe', 'safely' or 'safety' to identify articles on the adverse effects of medical devices than articles on drug effects. The wide disparity of potentially useful search terms and the lack of any one term successfully retrieving the majority of articles would suggest that any filter will need to be developed using a large set of relevant records to ensure that the optimal combination of search terms is captured.

The main difference between MEDLINE and Embase was the higher number of specific adverse effects terms presents in Embase. While this undoubtedly improves the sensitivity of Embase searches, it may be to the detriment of the precision of Embase searches. The trade-off between sensitivity and precision in database searching is inevitable, and any search filter development will need to consider this trade-off.

The relatively high proportion of records with specific named adverse effects in the abstract or in the indexing in Embase is reassuring given that adverse effects are often a secondary or even tertiary outcome in primary studies. Previous research in 2001 indicated that adverse drug effects terms are not present in the title, abstract or indexing nearly a quarter of all relevant research and this problem is more likely to be an issue in articles of non-drug interventions [8]. This finding led to recommendations that searchers should not rely on adverse effects terms [9]. However, since 2001, there have been improvements in the reporting of adverse effects in the title, abstract or indexing in primary studies related to drug interventions [4]. This may have resulted from efforts such as the CONSORT extension for harms [10], calls from the Cochrane Adverse Effects Methods Group (CAEMG) and in the published literature [11-33]. These improvements may extend to the reporting in articles on medical devices.

Interestingly, the results of this case study indicate that adverse effects terms are more likely to be present in the bibliographic records for medical devices than for drug interventions $[4,8]$. However, it should be recognised that this case study systematic review included many recent articles and improvements in adverse effects reporting have been identified over time [4]. It should also be noted that these results might also be affected by the level of caution demonstrated during the screening process in this case study systematic review and the same level of attention may not be present during routine screening of systematic review search results.

\section{Limitations}

This analysis is only based on one case study systematic review. This limits the generalisability of the results. This 
was an unusual review in that the authors were able to obtain unpublished data directly from the manufacturer. The included studies also included an unusually high number of conference abstracts and multiple publications for the same study (particularly for the clinical trials). The medical device is also rather atypical for a device in that it has a pharmaceutical component. Thus, our findings may be less generalizable.

Analysis of the precision of the search terms would have been of interest, however, was beyond the scope of this case study.

The usefulness of searching with specific terms is measured here, but this will only be applicable to those reviews in which the searchers have a clear idea beforehand what the potential key events are. This approach is not as useful for unexpected or new events. However, in this case study review, we have also demonstrated the value of generic adverse effects terms which can be used in any review. More research is required using more case study systematic reviews. Nevertheless, our evaluation is an important first step to guide systematic reviewers in an area which is likely to gain greater prominence, given the heightened public interest in safety of devices following recent scares.

\section{Conclusions}

This case study demonstrates the presence of adverse effects terms in the bibliographic records of articles on medical devices. The search terms most successful in identifying adverse effects of medical devices differed from those used in adverse effects search filters for drug interventions. Consequently, creating a search filter for adverse effects of medical devices is feasible and should be a research priority.

\section{Additional files}

Additional file 1: Table $\mathbf{S 1}$. Records retrieved by individual search terms in MEDLINE.

Additional file 2: Table S2. Records retrieved by individual search terms in Embase.

\section{Competing interests}

The authors declare that they have no competing interests.

\section{Authors' contributions}

SG conceived the idea, wrote the protocol, carried out the analysis and wrote the first and subsequent drafts of the paper. KW conducted the original searches in the systematic review and commented and contributed to all stages of the paper. MR carried out all stages in the original review, from sifting the records, data extraction and analysis. MR also helped ascertain the included studies for this paper and commented and contributed to all versions of the paper. All authors read and approved the final manuscript.

Received: 20 August 2014 Accepted: 24 September 2014

Published: 13 October 2014

\section{References}

1. Chou RAN, Atkinsc D, Ismailad AS, Santaguidad P, Smith DH, Whitlock E, Wilt TJ, Moher D: AHRQ Series Paper 4: assessing harms when comparing medical interventions: AHRQ and the effective health-care program. J Clin Epidemiol 2010, 63:502-512.

2. Golder SLY: Search strategies to identify information on adverse effects: a systematic review. J Med Libr Assoc 2009, 97(2):84-92.

3. Golder S, Loke YK: The performance of adverse effects search filters in MEDLINE and EMBASE. Health Inf Libr J 2012, 29(2):141-151.

4. Golder S, Loke YK: Failure or success of electronic search strategies to identify adverse effects data. J Med Libr Assoc 2012, 100(2):130-134.

5. Farrah KMU, Cimon MK: Playing it safe: validating search filters for adverse events. Poster presented at: 2013 Annual Meeting and Exhibition of the Medical Library Association Add to My Files (MLA '13), the 11th International Congress on Medical Librarianship (ICML), the 7th International Conference of Animal Health Information Specialists (ICAHIS), and the 6th International Clinical Librarian Conference (ICLC). One Health: Information in an Interdependent World; 2013 May 3-8. Boston, MA. Available from: http://www.cadth.ca/ media/is/MLA-Poster_Kelly.pdf.

6. Rodgers MABJ, Heirs MK, Higgins JP, Mannion RJ, Simmonds MC, Stewart $L A$ : Reporting of industry funded study outcome data: comparison of confidential and published data on the safety and effectiveness of rhBMP-2 for spinal fusion. BMJ 2013, 346:f3981.

7. Simmonds MBJ, Heirs M, Higgins JPT, Mannion R, Rodgers M, Stewart L: Safety and effectiveness of recombinant human bone morphogenetic protein-2 for spinal fusion: a meta-analysis of individual-participant data. Ann Intern Med 2013, 158(12):877-889.

8. Derry SLY, Aronson K: Incomplete evidence: the inadequacy of databases in tracing published adverse drug reactions in clinical trials. BMC Med Res Methodol 2001, 1:7.

9. Higgins JPT, Green S: Cochrane handbook for systematic reviews of interventions version 5.1.0 [updated March 2011]. The Cochrane Collaboration 2011. Available from: www.cochrane-handbook.org.

10. Ioannidis JP, Evans SJ, Gotzsche PC, O'Neill RT, Altman DG, Schulz K, Moher $D$ : Better reporting of harms in randomized trials: an extension of the CONSORT statement. Ann Intern Med 2004, 141(10):781-788.

11. Haidich AB, Birtsou C, Dardavessis T, Tirodimos I, Arvanitidou M: The quality of safety reporting in trials is still suboptimal: survey of major general medical journals. J Clin Epidemiol 2011, 64(2):124-135.

12. Yazici Y: Safety reporting in randomized clinical trials-a need for improvement. Bull NYU Hosp Jt Dis 2009, 67(2):209-210.

13. Papanikolaou PN, Churchill R, Wahlbeck K, loannidis JP: Safety reporting in randomized trials of mental health interventions. Am J Psychiatry 2004 161(9):1692-1697.

14. Ioannidis JP, Lau J: Improving safety reporting from randomised trials. Drug Saf 2002, 25(2):77-84.

15. Neidig JL, Koletar SL: Safety reporting in clinical trials. JAMA 2001, 285(16):2077-2078.

16. Kohl KS, Bonhoeffer J: Steering Committee of the Brighton C. Safety reporting in clinical trials. JAMA 2001, 285(16):2076-2077.

17. Loannidis JP, Lau J: Completeness of safety reporting in randomized trials: an evaluation of 7 medical areas. JAMA 2001, 285(4):437-443.

18. Chowers MY, Gottesman BS, Leibovici L, Pielmeier U, Andreassen S, Paul M: Reporting of adverse events in randomized controlled trials of highly active antiretroviral therapy: systematic review. J Antimicrob Chemother 2009, 64(2):239-250.

19. Yazici Y: Some concerns about adverse event reporting in randomized clinical trials. Bull NYU Hosp Jt Dis 2008, 66(2):143-145.

20. Cheng CW, Bian ZX, Li YP, Moher D, Wu TX, Dagenais S, Li J, Li TQ: Transparently reporting adverse effects of traditional Chinese medicine interventions in randomized controlled trials. Zhong Xi Yi Jie He Xue Bao 2008, 6(9):881-886.

21. Bauer KA, Hammerman S, Rapoport B, Lacouture ME: Completeness in the reporting of dermatologic adverse drug reactions associated with monoclonal antibody epidermal growth factor receptor inhibitors in phase II and III colorectal cancer clinical trials. Clin Colorectal Cancer 2008, 7(5):309-314. Research Support, Non-U.S. Gov't Review.

22. Woodworth T, Furst DE, Alten R, Bingham C, Yocum D, Sloan V, Tsuji W, Stevens R, Fries J, Witter J, Johnson K, Lassere M, Brooks P: Standardizing assessment and reporting of adverse effects in rheumatology clinical trials II: the Rheumatology Common Toxicity Criteria v.2.0. J Rheumatol 2007, 34(6):1401-1414. Consensus Development Conference. 
23. Nuovo J, Sather C: Reporting adverse events in randomized controlled trials. Pharmacoepidemiol Drug Saf 2007, 16(3):349-351.

24. Extermann M: More thoughts on the reporting of adverse events in cancer clinical trials. J Clin Oncol 2007, 25(7):918.

25. Burfeind DB: Streamlining adverse event reporting in trials. Dermatol Nurs 2006, 18(4):381-382

26. Anderson SJ: Some thoughts on the reporting of adverse events in phase II cancer clinical trials. J Clin Oncol 2006, 24(24):3821-3822.

27. Lassere MN, Johnson KR, Woodworth TG, Furst DE, Fries JF, Kirwan JR, Tugwell PS, Day RO, Brooks PM: Challenges and progress in adverse event ascertainment and reporting in clinical trials. J Rheumatol 2005, 32(10):2030-2032.

28. Trotti A, Bentzen SM: The need for adverse effects reporting standards in oncology clinical trials. J Clin Oncol 2004, 22(1):19-22.

29. Liauw WS, Day RO: Adverse event reporting in clinical trials: room for improvement. Med J Aust 2003, 179(8):426-428.

30. Martin PJ, Antin JH, Weisdorf DJ, Paton V, Horowitz MM: Reporting of adverse event data in hematopoietic stem cell transplantation clinical trials involving investigational new drugs or devices: a report from the William Guy Forbeck Foundation 2001 focus meeting on clinical trials in hematopoietic stem cell transplantation. Biol Blood Marrow Transplant 2002, 8(6):295-302

31. Loke YK, Derry S: Reporting of adverse drug reactions in randomised controlled trials-a systematic survey. BMC Clin Pharm/ 2001, 1:3.

32. Ethgen M, Boutron I, Steg PG, Roy C, Ravaud P: Reporting of harm in randomized controlled trials evaluating stents for percutaneous coronary intervention. Trials 2009, 10:29.

33. Ethgen M, Boutron I, Baron G, Giraudeau B, Sibilia J, Ravaud P: Reporting of harm in randomized, controlled trials of nonpharmacologic treatment for rheumatic disease. Ann Intern Med 2005, 143(1):20-25.

doi:10.1186/2046-4053-3-113

Cite this article as: Golder et al.: Failure or success of search strategies to identify adverse effects of medical devices: a feasibility study using a systematic review. Systematic Reviews 2014 3:113.

\section{Submit your next manuscript to BioMed Central and take full advantage of:}

- Convenient online submission

- Thorough peer review

- No space constraints or color figure charges

- Immediate publication on acceptance

- Inclusion in PubMed, CAS, Scopus and Google Scholar

- Research which is freely available for redistribution 